\title{
Reliability -based Sensitivity Intelligent Design of Capstan with Non-normal Distribution Parameters
}

\author{
Zhe Liu ${ }^{1, a^{*}}$, Fenghe Tao ${ }^{1, b}$ and Changzhi Jia ${ }^{1, c}$ \\ ${ }^{1}$ Department of Artillery Engineering, Ordnance Engineering College \\ Shijiazhuang, Hebei, 050003, P.R. China

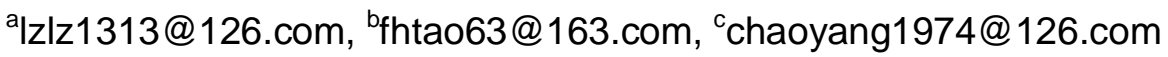

Keywords: Capstan; Reliability Sensitivity Design; Arbitrary Distribution Parameters; Fourth-moment Technique.

Abstract. Numerical method for reliability sensitivity design is presented based on fourth moment technique, and the reliability sensitivity of the capstan in track-laying vehicle with non-normal random parameters is extensively discussed. The variation regularities of reliability sensitivity are obtained and the effects of design parameters on reliability of the capstan in track-laying vehicle are studied. The method presented in this paper provides the theoretic basis for the reliability design of the capstan in track-laying vehicle, the production can be very useful if it extended to correlated area of machine sensitivity design.

\section{Introduction}

Reliability analysis of automobile components is highly related to the quality of product in terms of its service lifetime, maintenance cost, and the risk of failure. In engineering practice, given the probability distribution of basic modelling variables, reliability analysis determines the corresponding failure probability of the structure under consideration ${ }^{[1,2]}$. Inversely, it is also significant to calibriate the distribution parameters of modelling variables given an predefined level of structural failure probability. The inverse procedure of reliability analysis is referred to the reliability-based design optimization in the pape ${ }^{[3,4]}$. Since that available optimization procedures are usually fomulated as the gradient-based trade-off process, sensitivities of structural failure probability(Pf)with respect to each distributon parameter, then, are vital to implement the optimization. In addition, reliability-based sensitivity analysis is cosidered to be important due to the fact that it is capable of evaluating the impact of design parameter changes on component safety ${ }^{[5,6]}$.

This paper discusses the reliability-based analysis of capstan with non-normal distributions of parameters, based on the perturbation technique and the fourth-moment technique ${ }^{[7]}$. The paper is organized as follows. Section 1 reviews the developments of reliability-based analysis by using the methods of stochastic perturbation and first-four moments. In section 2, the reliability-sensitivity analysis is determined to measure the variations of structural reliability with respect to distribution parameters of input random variables. In section 3, numerical examples of on reliability-based sensitivity analysis on capstan are employed to illustrate the application of the proposed method. Finally, section 4 summarizes the conclusions.

\section{Perturbation Method of Reliability Design}

A fundamental problem in reliability analysis is the computation of the multi-fold integral of the reliability R:

$$
R=\int_{g(X)>0} f_{X}(X) d X
$$

Where $f_{X}(X)$ denotes the probability density function of random parameter vector $X=\left(X_{1} X_{2} \mathrm{~L} X_{n}\right)^{T}$, and $g(X)$ defines the state function, representing the safe and failure states where

$$
\left\{\begin{array}{c}
g(X) \leq 0, \text { failurestate, } \\
g(X)>0, \text { safestate, }
\end{array}\right.
$$


And $g(X)=0$ is the limit-state equation representing an n-dimensional surface, which may be called the "limit-state surface" or "failure surface".

The vector of random parameters $X$ and the state function $g(X)$ are expanded as

$$
\begin{gathered}
X=X_{d}+\varepsilon X_{p} \\
g(X)=g_{d}(X)+\varepsilon g_{p}(X)
\end{gathered}
$$

Where $\varepsilon$ is a small parameter. The part of Eqs. (3) and (4) that is denoted by subscript $d$ is the certain part of the random parameters, and the part that is denoted by subscript $\mathrm{p}$ is the random part, having a zero mean value in the random parameters. Obviously, it is necessary for the value of the random part to be smaller than the value of the certain part. Both sides of Eqs.(3) and (4) are evaluated about the mean value of the random variables as follows:

$$
\begin{gathered}
E(X)=\mathrm{E}\left(X_{d}\right)+\varepsilon E\left(X_{p}\right)=X_{d} \\
E[g(X)]=\mathrm{E}\left[\mathrm{g}\left(X_{d}\right)\right]+\varepsilon E\left[g\left(X_{p}\right)\right]=g_{d}(X)
\end{gathered}
$$

Both sides of Eqs.(3) and (4) are evaluated about the variance and third and fourth moments of the random variables, and the state function as follows:

$$
\begin{gathered}
E[g(X)]=\mathrm{E}\left[\mathrm{g}\left(X_{d}\right)\right]+\varepsilon E\left[g\left(X_{p}\right)\right]=g_{d}(X) \\
V \operatorname{ar}[g(X)]=E\left\{[g(X)-E g(X)]^{[2]}\right\}=\varepsilon^{2} E\left\{\left[g_{p}(X)\right]^{[2]}\right\} \\
C_{3}[g(X)]=E\left\{[g(X)-E g(X)]^{[3]}\right\}=\varepsilon^{3} E\left\{\left[g_{p}(X)\right]^{[3]}\right\} \\
C_{4}[g(X)]=E\left\{[g(X)-E g(X)]^{[4]}\right\}=\varepsilon^{4} E\left\{\left[g_{p}(X)\right]^{[4]}\right\}
\end{gathered}
$$

According to Kronecker algebra, the notation $(\cdot)^{[k]}$ represents the $k$ th order Kronecker power of an arbitrary matrix. i.e., for the arbitrary matrix $P$, its $k$ th Kronecker power can be expressed as $P^{[k]}=P \otimes P^{[k-1]}=P \otimes P \otimes \mathrm{L} P$. The symbol $\otimes$ represents the Kronecker product, which is defined as $(A)_{p \times q} \otimes(B)_{s \times t}=\left(a_{i j} B\right)_{p s \times q t}$.

The probability density function or joint probability density function of the basic random parameters is needed to calculate the structural reliability or failure probability. However, it is difficult in practice to have enough information to determine their distribution types. Even when the probability distribution of the state function is approximated, it is difficult to obtain failure probability by numerical integration ${ }^{[8]}$.

Thus, the moment-based method is one of the most practical methods for reliability analysis. In the second-moment method, the reliability can be defined as

$$
R_{S M} \approx \Phi\left(\beta_{S M}\right)
$$

Where

$$
\beta_{S M}=\frac{\mu_{g}}{\sigma_{g}}=\frac{E[g(X)]}{\sqrt{\operatorname{Var}[g(X)]}}
$$

and $\Phi(g)$ is the cumulative stand normal distribution function, $\mu_{g}$ is the mean value of the state function $g(X)$.

The Fourth-moment method can be used under the circumstance of the state function being explicit expression and the first four terms of the random parameters being known ${ }^{[9]}$. The limitation of the Fourth-moment method is that more moments should be known while comparing to the Second-moment method and thus statistical difficulties may occur. However, because of this 
limitation, the Fourth-moment method has the advantage when dealing with reliability problems with abnormal distribution functions.

Under the circumstance of the first four moments of state function and random variables being given, and the first four moments of the basic random parameters known, the reliability can be obtained by using the fourth-moment method:

$$
R_{F M} \approx \Phi\left(\beta_{F M}\right)
$$

where

$$
\beta_{F M}=\frac{3\left(\alpha_{4 g}-1\right) \beta_{S M}+\alpha_{3 g}\left(\beta_{S M}^{2}-1\right)}{\sqrt{\left(9 \alpha_{4 g}-5 \alpha_{3 g}^{2}-9\right)\left(\alpha_{4 g}-1\right)}}
$$

And $\alpha_{3 g} 、 \alpha_{4 g}$ is the coefficient of skewness and kurtosis,

$$
\alpha_{3 g}=\frac{E\left[\left(X-\mu_{g}\right)^{3}\right]}{\sigma_{g}^{3}}=\frac{\theta_{g}}{\sigma_{g}^{3}} \alpha_{4 g}=\frac{E\left[\left(X-\mu_{g}\right)^{4}\right]}{\sigma_{g}^{4}}=\frac{\eta_{g}}{\sigma_{g}^{4}}
$$

The computational relationship between the skewness and Kurtosis of arbitrary random variables in the state equations is $\alpha_{4 g} \geq \alpha_{3 g}^{2}+1$.

\section{Reliability Sensitivity}

The reliability sensitivity with respect to the mean value $X_{d}$ and variance $\operatorname{Var}(X)$ of the basic random parameters $X$ is derived as follows:

$$
\begin{aligned}
\frac{d R_{F M}\left(\beta_{F M}\right)}{d X_{d}^{T}} & =\frac{\partial R_{F M}\left(\beta_{F M}\right)}{\partial \beta_{F M}} \cdot \frac{\partial \beta_{F M}}{\partial \beta_{S M}} \cdot \frac{\partial \beta_{S M}}{\partial \mu_{g}} \cdot \frac{\partial \mu_{g}}{\partial X_{d}^{T}} \\
\frac{d R_{F M}}{d \operatorname{Var}(X)} & =\left[\frac{\partial R_{F M}}{\partial \beta_{S M}}\left(\frac{\partial \beta_{S M}}{\partial \beta_{S M}} \frac{\partial \beta_{S M}}{\partial \sigma_{g}}+\frac{\partial \beta_{S M}}{\partial \sigma_{g}}\right)\right] \frac{\partial \sigma_{g}}{\partial \operatorname{Var}(X)}
\end{aligned}
$$

The terms on the right side of Eqs. (16) and (17) can be expressed as

$$
\frac{\partial R_{F M}}{\partial \beta_{F M}}=\varphi\left(\beta_{F M}\right)
$$

where $\varphi(g)$ is the probability density function of standard normal distribution,

$$
\begin{gathered}
\frac{\partial \beta_{F M}}{\partial \beta_{S M}}=\frac{3\left(\alpha_{4 g}-1\right)+2 \alpha_{3 g} \beta_{S M}}{\sqrt{\left(9 \alpha_{4 g}-5 \alpha_{3 g}^{2}-9\right)\left(\alpha_{4 g}-1\right)}} \\
\frac{\partial \beta_{S M}}{\partial \mu_{g}}=\frac{1}{\sigma_{g}} \\
\frac{\partial \mu_{g}}{\partial X_{d}^{T}}=\left(\frac{\partial g}{\partial X_{d 1}} \frac{\partial g}{\partial X_{d 2}} \mathrm{~L} \frac{\partial g}{\partial X_{d 3}}\right) \\
\frac{\partial \beta_{S M}}{\partial \sigma_{g}}=-\frac{\mu_{g}}{\sigma_{g}^{2}} \\
\frac{\partial \sigma_{g}}{\partial \operatorname{Var}(X)}=\frac{1}{2 \sigma_{g}}\left(\frac{\partial g}{\partial X} \otimes \frac{\partial g}{\partial X}\right) \\
\frac{\partial \beta_{F M}}{\partial \sigma_{g}}=-\frac{1}{\sigma_{g}}\left\{\frac{12 \alpha_{4 g} \beta_{S M}}{\sqrt{Q M}}+3 U\right.
\end{gathered}
$$


where $Q=9 \alpha_{4 g}-5 \alpha_{3 g}^{2}-9$

$$
\begin{aligned}
M & =\alpha_{4 g}-1 \\
U & =\alpha_{3 g}\left(\beta_{S M}^{2}-1\right)
\end{aligned}
$$

Substituting Eqs. (18)-(24) into Eqs. (16) and (17), one will obtain the reliability sensitivity results ${ }^{[10]}$, $d R_{F M} / d X_{d}^{T}$ and $d R_{F M} / d \operatorname{Var}(X)$.

\section{Examples}

A capstan is an important power transmission drive system components, it can transfer the power from the side reducer to track wheel, which engages through the gear and track wheel, it can toggle track campaign, only to withstand the torque effect.

Typically, the reliability-based robust design of automobile

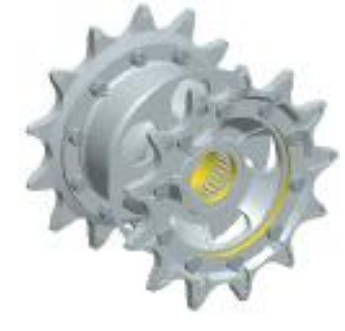

Fig. 1 Solid models of capstan

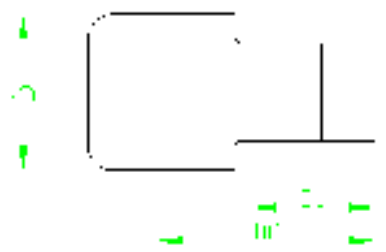

Fig.2 Structure sketch of a gear on capstan

The tensile stresses on the I-section of the capstan is

$$
\tau=\frac{3 P\left(D_{0}-D_{1}\right)}{\pi D_{1} h^{2}}
$$

where $P$ is the tensile load on the capstan, and the I-section dimensions are $h, D_{0}, D_{1}, P$.

From reliability theory, the state equation of the capstan is defined as

$$
g(X)=r-\tau
$$

where $r$ is the material strength. The random variable vector is given by $X=\left(r D_{0} D_{1} h P\right)^{T}$.

Generally speaking, dimensions and material properties are usually within normal distribution. The statistical characterization of material strength $r$ is $\left(\mu_{r}, \sigma_{r}\right)=(135,5.256) \mathrm{MPa}$. The dimensions are $\left(\mu_{h}, \sigma_{h}\right)=(50,0.25) \mathrm{mm},\left(\mu_{D_{0}}, \sigma_{D_{0}}\right)=(1200,6) \mathrm{mm},\left(\mu_{D_{1}}, \sigma_{D_{1}}\right)=(1000,5) \mathrm{mm}$. The load $P$ is an arbitrary distributed random variable, with first four moments.

$\left(\mu_{P}, \sigma_{P}, \theta_{P}, \eta_{P}\right)=\left(1.3025 \times 10^{6} \mathrm{~N}, 1.2024 \times 10^{5} \mathrm{~N}^{2}, 1.9872 \times 10^{15} \mathrm{~N}^{3}, 1.5724 \times 10^{21} N^{4}\right)$

Then, the reliability index, the reliability, and the reliability sensitivities can be determined as

$$
\begin{gathered}
\beta_{S M}=3.102912, \beta_{F M}=2.466713, R_{F M}=0.9931821, R_{M C S}=0.9935 \\
d R / d \bar{X}^{T}=\left[\begin{array}{c}
\partial R / \partial r \\
\partial R / \partial D_{0} \\
\partial R / \partial D_{1} \\
\partial R / \partial h \\
\partial R / \partial P
\end{array}\right]^{T}=\left[\begin{array}{c}
8.096 \times 10^{-4} \\
-5.586 \times 10^{-4} \\
6.737 \times 10^{-4} \\
4.709 \times 10^{-3} \\
-6.703 \times 10^{-8}
\end{array}\right]^{T}
\end{gathered}
$$

From the results obtained by reliability sensitivity method respectively, it can be found out that this method compared with MSC numerical simulation have a high accuracy, and the results from the sensitivity analysis, the thickness of the capstan with the highest sensitivity, the sensitivity of the load is minimum, so the need to control when design and manufacture of capstan, secondly, at the time of design and manufacturing parameters capstan main material strength should also be considered. With 
these parameter sensitivity analysis results, we can design and manufacture of intelligent mechanical structures have some help and guidance significance.

\section{Summary}

Based on the reliability of the study, this paper propose a method for calculating the reliability of the sensitivity of the capstan numerical methods, effective response to the various factors that affect their reliability capstan extent, if certain factors on the tracked vehicle parts failure have a greater impact, so in the process of design and manufacture of it needs to be strictly controlled, making it small enough to ensure that the changes in vehicle parts have adequate safety and reliability; on the contrary, if the change of a factor of vehicle reliability is not significantly affected parts, vehicle parts during the design and manufacturing process, can be treated as a fixed value, which can reduce the complexity of the design and manufacture.

The numerical method only needs to know the first four moments of random parameters for each component, the fourth moment approach for reliability sensitivity design of vehicle components is proposed, an assessment of influence of changes of determined design parameters on vehicle components'reliability is made to fully reflect the sensitivity of each design parameter to vehicle components'failure.

\section{References}

[1] LU Chun-mei, ZHANG Yi-min, LIU Yu, ZHOU Na, Sensitivity analysis for frequency reliability of a random bending vibration system of continuous beam, Journal of vibration and shock, Vol.32, No.18, 2013, pp. 159-162.

[2] HUANG Yi-min, LIU Wei, LIU Yong-shou, YUE Zhu-feng, Parameter sensitivity and resonance reliability of a fluid-filled pipeline. Journal of vibration and shock, Vol.29, No.1, 2010, pp. 193-195.

[3] LIU Yan-ming, ZHANG Xue-liang, YANG Bo, MATLAB-based Four Moment Method for Reliability and Optimization Design, Mechanical Engineering \& Automation, Vol.22, No.5, 2011, pp. 27-29.

[4] HE Xiang-dong, ZHANG Yi-min, WEN Bang-chun, Stable Reliability Sensitivity Design of Compressive Bar With Non-Normal Distribution Parameters, Journal of Astronautics, Vol.28, No.5, 2007, pp. 1401-1404.

[5] Zhang Yimin, Yang Zhou, Reliability-Based Sensitivity Analysis of Vehicle Components with Non-normal Distribution Parameters, International Journal of Automotive Technology, Vol.10, No.2, 2009, pp. 181-194.

[6] $\mathrm{Xu} \mathrm{H}$, Rahman S, Decomposition methods for structural reliability analysis, Probabilistic Engineering Mechanics, Vol.20, No.3, 2005, pp. 239 250.

[7] SONG Jun, LU Zhen-zhou, A New Reliability Sensitivity Analysis Method, Acta Aeronautica Et Astronautica Sinica, Vol.27, No.5, 2006, pp. 823-826.

[8] KIM K P, HUH H. Dynamic limit analysis formulation for impact simulation of structural members, Int.J.Solids\&Struc, Vol.43, No.21, 2006, pp. 6488 6501.

[9] SUES R H, CESARE M A, System reliability and sensitivity factors via the MPPSS method, Probab.Eng.Mech, Vol.20, No.2, 2005, pp. 148 157.

[10] GU L, YANG R J, On reliability-based optimisation methods for automotive structures, Int.J.Mater.\&Prod.Technol, Vol.25, No.1-3, 2006, pp. 3 26. 